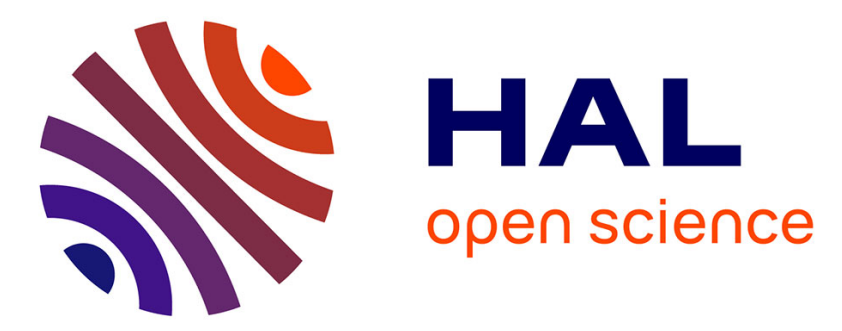

\title{
Sensitivity Analysis for an Incompressible Aeroelastic System
}

\author{
Miguel Angel Fernández, Marwan Moubachir
}

\section{To cite this version:}

Miguel Angel Fernández, Marwan Moubachir. Sensitivity Analysis for an Incompressible Aeroelastic System. [Research Report] RR-4264, INRIA. 2001. inria-00072323

\section{HAL Id: inria-00072323 \\ https://hal.inria.fr/inria-00072323}

Submitted on 23 May 2006

HAL is a multi-disciplinary open access archive for the deposit and dissemination of scientific research documents, whether they are published or not. The documents may come from teaching and research institutions in France or abroad, or from public or private research centers.
L'archive ouverte pluridisciplinaire HAL, est destinée au dépôt et à la diffusion de documents scientifiques de niveau recherche, publiés ou non, émanant des établissements d'enseignement et de recherche français ou étrangers, des laboratoires publics ou privés. 


\title{
Sensitivity analysis for an incompressible aeroelastic system
}

\author{
Miguel-Angel Fernández — Marwan Moubachir
}

\section{$N^{\circ} 4264$}

Septembre 2001

THÈME 4 



\title{
Sensitivity analysis for an incompressible aeroelastic system
}

\author{
Miguel-Angel Fernández*, Marwan Moubachir ${ }^{\dagger}$ \\ Thème 4 - Simulation et optimisation \\ de systèmes complexes \\ Projet MACS \\ Rapport de recherche $\mathrm{n}^{\circ} 4264$ - Septembre 2001 - 33 pages
}

\begin{abstract}
This article deals with problems arising in the sensitivity analysis for fluid-structure interaction systems. Our model consists of a fluid described by the incompressible Navier-Stokes equations interacting with a solid under large deformations. We obtain a linearized problem which allow us to compute the derivative of the state variable with respect to a given boundary parameter. We use a particular definition of the first-order correction for the perturbed state and consider a weak Arbitrary Euler-Lagrange formulation for the coupled system.
\end{abstract}

Key-words: Fluid-structure interaction, Navier-Stokes equations, ALE formulation, sensitivity analysis.

* INRIA-MACS, email: miguel.fernandez@inria.fr

† Laboratoire Central des Ponts et Chaussées - LMSGC, email:

marwan.moubachir@lcpc.fr 


\section{Analyse de sensibilité pour un système aéorélastique incompressible}

Résumé : Cet article a pour objet l'analyse de sensibilité pour un système mécanique constitué d'un solide élastique, dont le comportement est régi par les lois de l'élasticité non-linéaire, au sein d'un fluide newtonien en écoulement incompressible, dont l'évolution est régie par les équations de Navier-Stokes. On étudie les variations du système vis à vis d'une donnée de Dirichlet frontière associée à la vitesse du fluide. On recherche le système satisfait par la dérivée de l'état par rapport à cette donnée.

Mots-clés : Interaction fluide-structure, équations de Navier-Stokes, formulation ALE, analyse de sensibilité. 


\section{Introduction}

Nowadays sensitivity analysis has reached an important role in the design of complex industrial systems. The main issue consists in characterizing the response level of a mechanical system due to crucial parameters perturbations. This characterization is performed on the mathematical model deduced from the physical behavior of the system.

Most of the work has been done on systems described by partial differential equations in cylindrical domains, i.e in time independent domains. For example, in the last decades, complex fluid models has been analyzed on the theoretical and numerical aspects $[1,9,10,11,14]$. However, concerning the analysis of coupled systems written in non-cylindrical domains, few works have been realized [15, 17, 18, 20].

Nevertheless, thanks to high computing and numerical schemes performances increase, numerical simulations of such systems involved in aeroelasticity, biomechanics or civil engineering, have been successfully performed $[3,4,7,8,19]$.

Our work is an attempt towards design and control of systems involving fluid-structure coupling. Our contribution is focused on the derivation of the linearized problem satisfied by the state gradient, with respect to a Dirichlet boundary condition, where the state satisfies the coupled incompressible Navier-Stokes equations with the non-linear elastodynamic equations.

First of all, we introduce the mechanical problem and its mathematical description. We use the classical arbitrary Euler-Lagrange (ALE) formulation, particularly suited for problems involving moving boundaries. Then, in the second part, we state the main result of this article, namely the state gradient computation and its associated linearized problem. Finally, its proof is fully developed. We must point out that the proof is highly based on a particular definition for the state perturbations, which has been introduced before, in a different context, by Fanion-Fernández-Le Tallec [5]. Our result was already announced as a brief note [6].

$\mathrm{RR} \mathrm{n}^{\circ} 4264$ 


\section{Mechanical problem}

In this section we introduce a fluid-structure interaction model. We consider a solid located at time $t \geq 0$ in a domain $\Omega^{s}(t) \subset \mathbb{R}^{3}$ with boundary $\Gamma^{s}(t)$. It is surrounded by a fluid in $\mathbb{R}^{3}$. We introduce a control volume $\Omega \subset \mathbb{R}^{3}$ containing the solid at each time $t \geq 0$. The notation $\partial \Omega$ stands for the boundary of $\Omega$. Hence, the fluid evolution is restricted to the domain $\Omega^{f}(t)=\Omega-\bar{\Omega}^{s}(t)$. In the sequel we set $\Gamma^{f}=\partial \Omega$ and

$$
\partial \Omega^{f}(t)=\Gamma^{f} \cup \Gamma^{s}(t)
$$

stands for the fluid domain boundary, see figure 1.

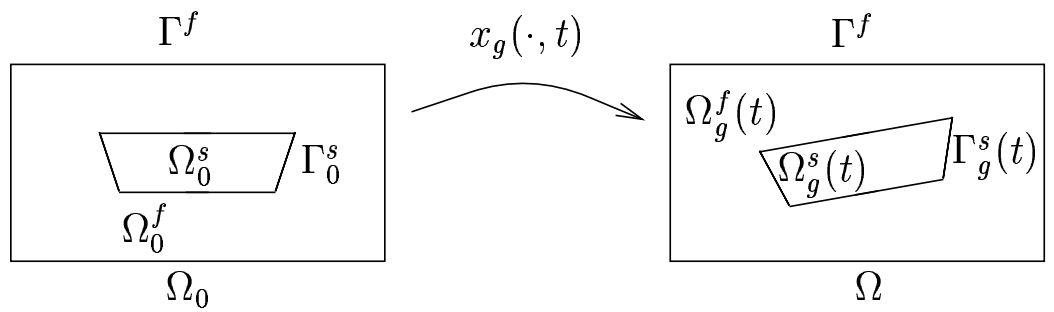

Figure 1: Geometric description

We assume the fluid to be newtonian viscous, homogeneous and incompressible. Its behavior is described by its velocity and pressure. The elastic solid under large displacements is described by its velocity and its stress tensor. The classical conservation laws of the continuum mechanics drive the evolution of these unknowns.

The fluid state satisfies the following incompressible Navier-Stokes equations written in eulerian conservative formulation:

$$
\begin{array}{r}
\frac{\partial u}{\partial t}+\operatorname{div}[u \otimes u-\sigma(u, p)]=0, \text { in } \Omega^{f}(t), \\
\operatorname{div} u=0, \text { in } \Omega^{f}(t),
\end{array}
$$


where $(u, p)$ stand, respectively, for the fluid velocity and pressure. In addition, the fluid stress tensor is given by

$$
\sigma(u, p)=-p \mathrm{I}+\nu\left[\nabla u+(\nabla u)^{T}\right],
$$

with $\nu$ the kinematic viscosity of the fluid.

Remark 1 In the sequel $\frac{\partial}{\partial t}{ }_{\mid a}$ stand for the time derivative operator keeping the space variable " $a$ " fixed.

In a fluid-structure interaction framework the evolution of the fluid domain $\Omega^{f}(t)$ is induced by the structural deformation through the fluid-structure interface $\Gamma^{s}(t)$. Indeed, by definition $\Omega^{f}(t)=\Omega-\bar{\Omega}^{s}(t)$. It leads us to describe $\Omega^{f}(t)$ according to a map acting in a fixed reference domain. This approach is usually used for the solid domain $\Omega^{s}(t)$, by means of the lagrangian formulation [12].

Given a material reference configuration for the solid $\Omega_{0}^{s} \subset \Omega$ with boundary $\Gamma_{0}^{s}$, we take $\Omega_{0}^{f}=\Omega-\bar{\Omega}_{0}^{s}$ as the reference fluid configuration. Then, the control volume $\Omega=\Omega^{f}(t) \cup \bar{\Omega}^{s}(t)$ is described by a smooth and injective map:

$$
\begin{aligned}
x: \bar{\Omega} \times \mathbb{R}^{+} & \longrightarrow \\
\left(x_{0}, t\right) & \longmapsto x=x\left(x_{0}, t\right) .
\end{aligned}
$$

We set $x^{f}=x_{\mid \Omega_{0}^{f}}$ and $x^{s}=x_{\mid \Omega_{0}^{s}}$, such that [13]:

- for $x_{0} \in \Omega_{0}^{s}, x^{s}\left(x_{0}, t\right)$ represents the position at time $t \geq 0$ of the material point $x_{0}$. This corresponds to the classical lagrangian flow,

- the map $x^{f}$ is defined from $x_{\mid \Gamma_{0}^{s}}^{s}$, as an arbitrary extension over domain $\bar{\Omega}_{0}^{f}$, which preserves $\Gamma_{0}^{f}=\Gamma^{f}=\partial \Omega$.

In short, the ALE map $x$ is given by

$$
\begin{aligned}
& x\left(x_{0}, t\right)=\operatorname{Ext}\left(\left.x^{s}\right|_{0} ^{s}\right)\left(x_{0}, t\right), \quad \forall x_{0} \in \bar{\Omega}_{0}^{f}, \\
& x\left(x_{0}, t\right)=x^{s}\left(x_{0}, t\right), \quad \forall x_{0} \in \bar{\Omega}_{0}^{s} .
\end{aligned}
$$

Here, Ext represents an extension operator from $\Gamma_{0}^{s}$ to $\bar{\Omega}_{0}^{f}$ such that

$$
\operatorname{Ext}\left(x^{s} \Gamma_{0}^{s}\right)_{\mid \Gamma_{0}^{f}}=I_{\Gamma_{0}^{f}}
$$

$\mathrm{RR} \mathrm{n}^{\circ} 4264$ 
Remark 2 We set $\Omega_{0}=\Omega_{0}^{f} \cup \bar{\Omega}_{0}^{s}$ as the reference domain, and $\Omega=\Omega^{f}(t) \cup$ $\bar{\Omega}^{s}(t)$ stands for the actual configuration at time $t>0$.

Remark 3 The operator Ext is arbitrary defined inside $\Omega_{0}^{f}$.

This map allows us to transport the fluid equations 1 inside the reference domain $\Omega_{0}^{f}$, yielding to the classical incompressible Navier-Stokes equations written in ALE conservative formulation [13], satisfied by $u: \Omega_{0}^{f} \times \mathbb{R}^{+} \longrightarrow \mathbb{R}^{3}$ and $p_{0}: \Omega_{0}^{f} \times \mathbb{R}^{+} \longrightarrow \mathbb{R}:$

$$
\begin{aligned}
\frac{\partial J u}{\partial t} \mid x_{0}+\operatorname{div}_{0}\left\{J[u \otimes(u-w)-\sigma(u, p)] F^{-T}\right\} & =0, \text { in } \Omega_{0}^{f}, \\
\operatorname{div}\left(J u F^{-T}\right) & =0, \text { in } \Omega_{0}^{f},
\end{aligned}
$$

where the quantities $F, J, w$ are defined by:

$$
\begin{aligned}
F & =\nabla_{0} x=\frac{\partial x}{\partial x_{0}} \\
J & =\operatorname{det}(F)>0 \\
w & =\left.\frac{\partial x}{\partial t}\right|_{x_{0}}
\end{aligned}
$$

Remark 4 From the definition of $x, w_{\mid \Omega_{0}^{s}}$ represents the solid velocity, whereas $w_{\mid \Omega_{0}^{f}}$ stands for the fluid control volume velocity, which usually differs from the fluid velocity inside $\Omega^{f}(t)$.

\subsection{Coupled strong ALE formulation}

The solid evolution is given by its motion $x^{s}$ and the stress tensor field $S$ (second Piola-Kirchoff tensor [2]). The field $S$ is related to $x^{s}$ through an appropriate constitutive law. Then, the pair $\left(x^{s}, S\right)$ satisfies the non-linear elastodynamic equations [2]:

$$
\ddot{x^{s}}-\operatorname{div}_{0}(F S)=f, \quad \text { in } \quad \Omega_{0}^{s} .
$$


The coupling between the solid and the fluid is realized through standard boundary conditions at the fluid-structure interface $\Gamma_{0}^{s}$, namely, the kinematic continuity of the velocity and the kinetic continuity of the stress [13]:

$$
\begin{array}{rll}
u=\dot{x}^{s}, & \text { on } & \Gamma_{0}^{s}, \\
F S n_{0}=J \sigma(u, p) F^{-T} n_{0}, & \text { on } & \Gamma_{0}^{s},
\end{array}
$$

where $n_{0}$ stands for the unit normal vector on $\Gamma_{0}^{s}$ pointing inside $\Omega_{0}^{s}$. Moreover, we endow the fluid equations with Dirichlet boundary condition on the far-field boundary $\Gamma_{0}^{f}$ :

$$
u=g, \quad \text { on } \quad \Gamma_{0}^{f} .
$$

In summary, the strong coupled problem, with a ALE formulation for the fluid, is given by:

$$
\begin{aligned}
& \frac{\partial J u}{\partial t}_{\mid x_{0}}+\operatorname{div}_{0}\left\{J[u \otimes(u-w)-\sigma(u, p)] F^{-T}\right\}=0, \quad \text { in } \quad \Omega_{0}^{f}, \\
& \operatorname{div}\left(J u F^{-T}\right)=0, \text { in } \Omega_{0}^{f}, \\
& u=g, \text { on } \Gamma_{0}^{f}, \\
& u=\dot{x^{s}}, \quad \text { on } \Gamma_{0}^{s}, \\
& F S n_{0}=J \sigma(u, p) F^{-T} n_{0}, \quad \text { on } \quad \Gamma_{0}^{s}, \\
& \ddot{x^{s}}-\operatorname{div}_{0}(F S)=f, \quad \text { in } \quad \Omega_{0}^{s}, \\
& \left(u, x^{s}, \dot{x}^{s}\right)_{\mid t=0}=\left(u_{0}, x^{s, 0}, x^{s, 1}\right), \quad \text { in } \quad \Omega_{0}^{f} \times\left(\Omega_{0}^{s}\right)^{2} .
\end{aligned}
$$

Here, $f$ represents the applied body force, $u_{0}$ the initial fluid velocity and $x^{s, 0}$, $x^{s, 1}$ the initial displacement and solid velocity, respectively.

\section{Sensitivity analysis}

In this section, we are dealing with the sensitivity analysis of the previous coupled system with respect to perturbations induced by the Dirichlet bound-

$\mathrm{RR} \mathrm{n}^{\circ} 4264$ 
ary condition $g: \Gamma_{0}^{f} \times \mathbb{R}^{+} \longrightarrow \mathbb{R}^{3}$. We are looking for the linearized system satisfied by the state gradient with respect to $g$.

Theorem 3.1 (Main result) Given a smooth state $\left(u_{g}, p_{g}, x_{g}^{s}\right)$ satisfying the coupled system (5) associated to the boundary condition $g$, the state derivative with respect to $g$ in the perturbation direction $\delta g: \Gamma_{0}^{f} \times \mathbb{R}^{+} \longrightarrow \mathbb{R}^{3}$ is given by the following expressions:

$$
\begin{aligned}
& \frac{D u_{g}}{D g}=\delta u+\nabla u_{g} \delta x^{f}, \quad \text { in } \quad \Omega_{g}^{f}(t) \\
& \frac{D p_{g}}{D g}=\delta p+\nabla p_{g} \delta x^{f}, \quad \text { in } \quad \Omega_{g}^{f}(t) \\
& \frac{D x_{g}^{s}}{D g}=\delta x^{s}, \quad \text { in } \quad \Omega_{0}^{s},
\end{aligned}
$$

where

$$
\delta x^{f}=\operatorname{Ext}^{\prime}\left(x_{g \mid \Gamma_{0}^{s}}^{s}\right) \delta x_{\mid \Gamma_{0}^{s}}^{s},
$$

and $\left(\delta u, \delta p, \delta x^{s}\right)$ satisfies the following linear coupled system:

$$
\begin{aligned}
& {\left.\frac{\partial \delta u}{\partial t}\right|_{g}}+\operatorname{div}\left(u_{g} \otimes \delta u+\delta u \otimes u_{g}-\delta \sigma\right)=0, \quad \text { in } \quad \Omega_{g}^{f}(t) \\
& \operatorname{div} \delta u=0, \quad \text { in } \Omega_{g}^{f}(t) \\
& \delta u=\delta g \quad \text { on } \quad \Gamma_{0}^{f} \\
& \delta u=\dot{\delta \dot{x}^{s}}-\nabla u_{g} \delta x^{s}, \quad \text { on } \quad \Gamma_{g}^{s}(t) \\
& \ddot{\delta x^{s}}-\operatorname{div}_{0}\left[F_{g} \delta S+\nabla_{0} \delta x^{s} S_{g}\right]=0, \quad \text { in } \Omega_{0}^{s}, \\
& {\left[F_{g} \delta S+\nabla_{0} \delta x S_{g}\right] n_{0}=J_{g}\left\|F_{g}^{-T} n_{0}\right\|\left(-\sigma_{g} \eta\left(\delta x^{s}\right)\right.} \\
& \left.+\nabla \sigma_{g} \delta x^{s} n_{g}+\delta \sigma n_{g}\right), \quad \text { on } \Gamma_{0}^{s} \\
& \left(\delta u, \delta x^{s}, \dot{\delta x^{s}}\right)_{\mid t=0}=0, \quad \text { in } \Omega_{g}^{f}(0) \times\left(\Omega_{0}^{s}\right)^{2} .
\end{aligned}
$$


with

$$
\begin{aligned}
\eta\left(\delta x^{s}\right) & =-\left[\operatorname{Idiv} \delta x^{s}-\left(\nabla \delta x^{s}\right)^{T}\right] n_{g}, & n_{g} & =\frac{F_{g}^{-T} n_{0}}{\left\|F_{g}^{-T} n_{0}\right\|} \\
\delta \sigma & =-\delta p \mathrm{I}+\nu\left(\nabla \delta u+\nabla \delta u^{T}\right), & \delta S & =S^{\prime}\left(x_{g}^{s}\right) \delta x^{s}
\end{aligned}
$$

Remark 5 The linear system satisfied by the variations $\left(\delta u, \delta p, \delta x^{s}\right)$ is posed in the moving domain $\Omega_{g}^{f}(t)$ provided by the reference state $\left(u_{g}, p_{g}, x_{g}^{s}\right)$.

Remark 6 The linearized problem consists of two subproblems posed in $\Omega_{g}^{f}(t)$ and $\Omega_{0}^{s}$, respectively, and coupled through non-standard boundary conditions on $\Gamma_{g}^{f}(t)$.

Remark 7 The fundamental property of the main result lies in the cancellation of the unknown $\delta x^{f}$ inside the equations satisfied by $\delta u$ in $\Omega_{g}^{f}(t)$. Thus, this will allow decoupling strategies at the fluid-structure interface and staggered procedures within a numerical approximation framework.

\section{Proof of the main result}

In this part we will present the detailed proof of our main result. We first introduce a weak formulation for the coupled system (5) and, giving a specific definition of the perturbations, we perform the linearization process.

\subsection{Weak formulation}

In this section, we introduce functional spaces which proof appropriate for the following computations. As a matter of fact, it is not, in any case, a mathematical optimal framework.

We consider fluid and solid test functions $\left(\hat{v}^{s}, \hat{v}^{f}\right) \in \mathcal{C}_{0}^{2}\left(\Omega_{0}\right)^{3} \times \mathcal{C}_{0}^{2}(\Omega)^{4}$, where $\mathcal{C}_{0}^{2}(\Omega)$ stands for the set of twice continuously differentiable functions in $\Omega$ with compact support.

Using the multiplication of test functions against the ALE equations (5), integrations by parts and taking into account boundary conditions, we obtain

$\mathrm{RR} \mathrm{n}^{\circ} 4264$ 
the following formulation:

$$
\begin{aligned}
& \int_{\Omega_{0}^{f}} \frac{\partial J_{g} u_{g}}{\partial t} \cdot \hat{x}_{0}^{f} \mathrm{~d} x_{0}-\int_{\Omega_{g}^{f}(t)}\left[\phi\left(u_{g}, \sigma_{g}\right)-\mathrm{I}_{1} u_{g} \otimes w_{g}\right]: \nabla \hat{v}^{f} \mathrm{~d} x_{g} \\
& +\int_{\Gamma_{g}^{s}(t)} \omega_{g} \cdot n_{g} \hat{v}_{2}^{f} \mathrm{~d} a_{g}+\int_{\Gamma_{0}^{s}} J_{g} \sigma_{g} F_{g}^{-T} n_{0} \cdot\left(\hat{v}^{s}-\hat{v}_{1}^{f}\right) \mathrm{d} a_{0}+\int_{\Omega_{0}^{s}} \ddot{x_{g}^{s}} \cdot \hat{v}^{s} \mathrm{~d} x_{0} \\
& +\int_{\Omega_{0}^{s}} F_{g} S_{g}: \nabla_{0} \hat{v}^{s} \mathrm{~d} x_{0}=\int_{\Omega_{0}^{s}} f \cdot \hat{v}^{s} \mathrm{~d} a_{0}, \quad \forall \hat{v}=\left(\hat{v}^{s}, \hat{v}^{f}\right) \in \mathcal{C}_{0}^{2}\left(\Omega_{0}\right)^{3} \times \mathcal{C}_{0}^{2}(\Omega)^{4},
\end{aligned}
$$

where

$$
\begin{aligned}
& \phi\left(u_{g}, \sigma_{g}\right)=\mathrm{I}_{1}\left(u_{g} \otimes u_{g}-\sigma_{g}\right)+\mathrm{I}_{2} \otimes u_{g}, \quad \mathrm{I}_{1}=\left(\begin{array}{ccc}
1 & 0 & 0 \\
0 & 1 & 0 \\
0 & 0 & 1 \\
0 & 0 & 0
\end{array}\right), \quad \mathrm{I}_{2}=\left(\begin{array}{l}
0 \\
0 \\
0 \\
1
\end{array}\right), \\
& \hat{v}^{f}=\left(\begin{array}{c}
\hat{v}_{1}^{f} \\
\hat{v}_{2}^{f}
\end{array}\right): \Omega \longrightarrow \mathbb{R}^{4}, \quad F_{g}=\nabla_{0} x_{g}, \quad x_{g}^{f}=\operatorname{Ext}\left(x_{g \mid \Gamma_{0}^{s}}^{s}\right), \quad w_{g}=\dot{x}_{g}{ }^{f} .
\end{aligned}
$$

We deal with Dirichlet conditions in a strong way:

$$
\begin{aligned}
& u_{g}=g, \quad \text { on } \quad \Gamma_{0}^{f}, \\
& u_{g}=\dot{x}_{g}^{s}, \quad \text { on } \Gamma_{0}^{s} .
\end{aligned}
$$

Remark 8 We must point out that we have chosen discontinuous test functions through the fluid-structure interface in order to derive boundary conditions for the linearized problem in a natural way.

The main difficulty while computing the state derivatives of (6) consists in defining the linear problem satisfied by the fluctuations $(\delta u, \delta p, \delta x)$. 
Perturbations $\delta g: \Gamma_{0}^{f} \times \mathbb{R} \longrightarrow \mathbb{R}^{3}$ in the Dirichlet boundary conditions lead to a new state $\left(u_{g+\delta g}, p_{g+\delta g}, x_{g+\delta g}\right)$, which satisfies a problem similar to (8):

$$
\begin{aligned}
& \int_{\Omega_{0}^{f}} \frac{\partial J_{g+\delta g} u_{g+\delta g}}{\partial t} \mid x_{0} \cdot \hat{v}_{1}^{f} \mathrm{~d} x_{0} \\
& -\int_{\Omega_{g+\delta g}^{f}(t)}\left[\phi\left(u_{g+\delta g}, \sigma_{g+\delta g}\right)-\mathrm{I}_{1} u_{g+\delta g} \otimes w_{g+\delta g}\right]: \nabla \hat{v}^{f} \mathrm{~d} x_{g+\delta g} \\
& +\int_{\Gamma_{g+\delta g}^{s}(t)} \omega_{g+\delta g} \cdot n_{g+\delta g} \hat{v}_{2}^{f} \mathrm{~d} a_{g+\delta g}+\int_{\Gamma_{0}^{s}} J_{g+\delta g} \sigma_{g+\delta g} F_{g+\delta g}^{-T} n_{0} \cdot\left(\hat{v}^{s}-\hat{v}_{1}^{f}\right) \mathrm{d} a_{0} \\
& +\int_{\Omega_{0}^{s}} \ddot{x}_{g+\delta g}^{s} \cdot \hat{v}^{s} \mathrm{~d} x_{0}+\int_{\Omega_{0}^{s}} F_{g+\delta g} S_{g+\delta g}: \nabla_{0} \hat{v}^{s} \mathrm{~d} x_{0} \\
& =\int_{\Omega_{0}^{s}} f \cdot \hat{v}^{s} \mathrm{~d} a_{0}, \quad \forall \hat{v}=\left(\hat{v}^{s}, \hat{v}^{f}\right) \in \mathcal{C}_{0}^{2}\left(\Omega_{0}\right)^{3} \times \mathcal{C}_{0}^{2}(\Omega)^{4}
\end{aligned}
$$

with

$$
\begin{aligned}
& u_{g+\delta g}=g+\delta g, \quad \text { on } \Gamma_{0}^{f}, \\
& u_{g+\delta g}=\dot{x}_{g+\delta g}^{s}, \quad \text { on } \Gamma_{0}^{s} .
\end{aligned}
$$

We are interested in the linearization of the perturbed problem around the reference state $\left(u_{g}, p_{g}, x_{g}\right)$. The crucial hypothesis is to assume that the state is differentiable with respect to Dirichlet boundary condition, i.e that $(\delta u, \delta p, \delta x)$ and $\delta g$ are equivalent in a given norm.

The variations $(\delta u, \delta p, \delta x)$ are defined in $\Omega_{g}$ as follow: for $x \in \Omega_{g}$ and $x_{0} \in \Omega_{0}$ such that $x=x_{g}\left(x_{0}, t\right)$, we define

$$
\delta x(x, t)=x_{g+\delta g}\left(x_{0}, t\right)-x_{g}\left(x_{0}, t\right),
$$

and

$$
\begin{aligned}
& \delta u(x, t)=u_{g+\delta g}(x+\delta x(x, t), t)-u_{g}(x, t)-\nabla u_{g}(x, t) \delta x(x, t), \\
& \delta p(x, t)=p_{g+\delta g}(x+\delta x(x, t), t)-p_{g}(x, t)-\nabla p_{g}(x, t) \delta x(x, t) .
\end{aligned}
$$

where $x \in \Omega_{g}^{f}(t)$ and $x_{0} \in \Omega_{0}^{f}$. In other words,

$$
\begin{aligned}
x_{g+\delta g} & =x_{g}+\delta x, \quad \text { in } \Omega_{0}, \\
u_{g+\delta g}\left(I_{\Omega_{g}^{f}(t)}+\delta x\right) & =u_{g}+\nabla u_{g} \delta x+\delta u, \quad \text { in } \Omega_{g}^{f}(t), \\
p_{g+\delta g}\left(I_{\Omega_{g}^{f}(t)}+\delta x\right) & =p_{g}+\nabla p_{g} \delta x+\delta p, \quad \text { in } \Omega_{g}^{f}(t) .
\end{aligned}
$$

$\mathrm{RR} \mathrm{n}^{\circ} 4264$ 
We must point out that the variations are defined through the fluctuations of the state evaluated at a given material point. Then $u_{g}+\nabla u_{g} \delta x+\delta u$ is considered as the first order approximation of $u_{g}\left(I_{\Omega_{g}^{f}(t)}+\delta x\right)$. Thus, in the next section, we perform a transport inside the reference problem (8).

\subsection{Transported reference problem}

Lemma 1 Given $\mathcal{V}_{0}$ a fixed bounded domain and $x: \mathcal{V}_{0} \longrightarrow \mathbb{R}^{3}$ an injective map, of regularity $\mathcal{C}^{2}\left(\mathcal{V}_{0}\right)^{3}$, we set $\mathcal{V}=x\left(\mathcal{V}_{0}\right)$. Let

$$
\Phi: \mathbb{R}^{3} \times \mathbb{R}^{3 \times 3} \times \mathbb{R}^{3} \longrightarrow \mathbb{R}^{4 \times 3}
$$

be a map of regularity $\mathcal{C}^{1}$, and $h \in L^{2}\left(\mathcal{V}_{0}\right)^{4}$. For any displacement $\delta x \in \mathcal{C}^{1}(\mathcal{V})^{3}$ and every element $(u, \sigma, w) \in \mathcal{C}^{1}(\mathcal{V})^{3 \times 9 \times 3}$ satisfying

$$
\int_{\mathcal{V}_{0}} h \cdot \hat{q} \mathrm{~d} x_{0}-\int_{\mathcal{V}} \Phi(u, \sigma, w): \nabla \hat{q} \mathrm{~d} x=0, \quad \forall \hat{q} \in \mathcal{C}_{0}^{2}(\mathcal{V})^{4},
$$

then $(u, \sigma, w)$ satisfies the following transported convected problem:

$$
\begin{aligned}
& \int_{\mathcal{V}_{0}} h \cdot(\nabla \hat{q} \delta x) \mathrm{d} x_{0}+\int_{\mathcal{V}}\left[\Phi(u, \sigma, w)\left(\operatorname{Idiv} \delta x-\nabla \delta x^{T}\right)\right. \\
& \left.+D_{(u, \sigma, w)} \Phi(u, \sigma, w)(\nabla u \delta x, \nabla \sigma \delta x, \nabla w \delta x)\right]: \nabla \hat{q} \mathrm{~d} x=0, \quad \forall \hat{q} \in C_{0}^{2}(\mathcal{V})^{4} .
\end{aligned}
$$

Where:

$$
\begin{aligned}
D_{(u, \sigma, w)} \Phi(u, \sigma, w)(\nabla u \delta x, \nabla \sigma \delta x & , \nabla w \delta x)=\frac{\partial \Phi}{\partial u}(u, \sigma, w)(\nabla u \delta x) \\
+ & \frac{\partial \Phi}{\partial \sigma}(u, \sigma, w)(\nabla \sigma \delta x)+\frac{\partial \Phi}{\partial w}(u, \sigma, w)(\nabla w \delta x) .
\end{aligned}
$$

Proof. We use here a similar framework introduced in Fanion-Fernández-Le Tallec [5]. Let $\hat{q} \in C_{0}^{2}(\mathcal{V})^{4}$ be a fixed test function. We set $K=\operatorname{supp}(\hat{q}) \subset \mathcal{V}$. For any $\varepsilon>0$, we define $x^{\varepsilon}=I_{\mathcal{V}}+\epsilon \delta x$. Using continuity of the map $x^{\varepsilon}$, it is possible to build a compact set $K_{0} \subset \mathcal{V}$ and a real number $\varepsilon_{0}>0$, such that for every $0<\varepsilon<\varepsilon_{0}, x^{\varepsilon}$ is injective and

$$
Q^{\varepsilon}=\left(x^{\varepsilon}\right)^{-1}(K) \subset K_{0}, \quad x^{\varepsilon}\left(K_{0}\right) \subset \mathcal{V},
$$




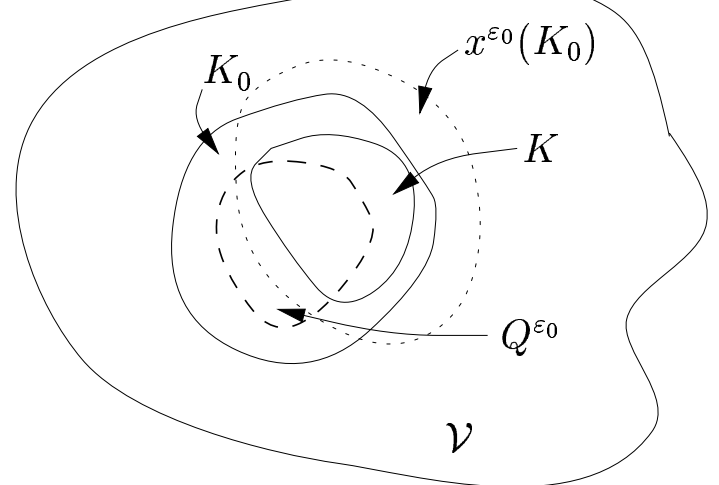

Figure 2: Sets description

see figure 2.

From (13) and using a change of variables, we get

$$
\begin{aligned}
0 & =\int_{\mathcal{V}_{0}} h \cdot \hat{q} \mathrm{~d} x_{0}-\int_{K} \Phi(u, \sigma, w): \nabla \hat{q} \mathrm{~d} x \\
& =\int_{\mathcal{V}_{0}} h \cdot \hat{q} \mathrm{~d} x_{0}-\int_{x^{\varepsilon}\left(Q^{\varepsilon}\right)} \Phi(u, \sigma, w): \nabla_{\varepsilon} \hat{q} \mathrm{~d} x^{\varepsilon} \\
& =\int_{\mathcal{V}_{0}} h \cdot \hat{q} \mathrm{~d} x_{0}-\int_{Q^{\varepsilon}} \Phi\left(u\left(x^{\varepsilon}\right), \sigma\left(x^{\varepsilon}\right), w\left(x^{\varepsilon}\right)\right):\left(\nabla_{\varepsilon} \hat{q}\right)\left(x^{\varepsilon}\right) \operatorname{det} \nabla x^{\varepsilon} \mathrm{d} x \\
= & \int_{\mathcal{V}_{0}} h \cdot \hat{q} \mathrm{~d} x_{0}-\int_{K_{0}}\left[\Phi\left(u\left(I_{\mathcal{V}}+\epsilon \delta x\right), \sigma\left(I_{\mathcal{V}}+\epsilon \delta x\right), w\left(I_{\mathcal{V}}+\epsilon \delta x\right)\right)\right. \\
& \left.\nabla\left(I_{\mathcal{V}}+\epsilon \delta x\right)^{-T}\right]: \nabla \hat{q}\left(I_{\mathcal{V}}+\epsilon \delta x\right) \operatorname{det} \nabla\left(I_{\mathcal{V}}+\epsilon \delta x\right) \mathrm{d} x .
\end{aligned}
$$

The last equality comes from the fact that $\hat{q}\left(I_{\mathcal{V}}+\epsilon \delta x\right)$ is zero outside of $Q^{\varepsilon} \subset$ $K_{0}$. Using a Taylor expansion of first order in $\varepsilon$, we obtain the following

$\mathrm{RR} \mathrm{n}^{\circ} 4264$ 
identity:

$$
\begin{aligned}
0= & \int_{\mathcal{V}_{0}} h \cdot \hat{q} \mathrm{~d} x_{0}-\int_{K_{0}} \Phi(u, \sigma, w): \nabla \hat{q} \mathrm{~d} x-\varepsilon \int_{K_{0}}\left[\Phi(u, \sigma, w)\left(\operatorname{Idiv} \delta x-\nabla \delta x^{T}\right)\right. \\
& \left.+D_{(u, \sigma, w)} \Phi(u, \sigma, w)(\nabla u \delta x, \nabla \sigma \delta x, \nabla w \delta x)\right]: \nabla \hat{q} \mathrm{~d} x \\
& -\varepsilon \int_{K_{0}} \Phi(u, \sigma, w) \nabla(\nabla \hat{q} \delta x) \mathrm{d} x+o(\varepsilon) .
\end{aligned}
$$

In the last equality, the first two terms are zero thanks to (13), and the last can be reduced to

$$
\int_{\mathcal{V}_{0}} h \cdot(\nabla \hat{q} \delta x) \mathrm{d} x
$$

using $\nabla \hat{q} \delta x$ as a function test in (13).

As a consequence, for $0<\varepsilon<\varepsilon_{0}$, the following equality holds true:

$$
\begin{aligned}
& 0=\varepsilon\left[\int_{K_{0}} \Phi(u, \sigma, w)\left(\mathrm{I} \operatorname{div} \delta x-\nabla \delta x^{T}\right)\right. \\
& \left.+D_{(u, \sigma, w)} \Phi(u, \sigma, w)(\nabla u \delta x, \nabla \sigma \delta x, \nabla w \delta x): \nabla \hat{q} \mathrm{~d} x-\int_{\mathcal{V}_{0}} h \cdot(\nabla \hat{q} \delta x) \mathrm{d} x\right]+o(\varepsilon) .
\end{aligned}
$$

By simply dividing by $\varepsilon$ and letting $\varepsilon$ tend to zero, we get (14).

We shall need later on the following corollary:

Corollary 4.1 Under the hypothesis of Lemma 1, with $\delta x \in \mathcal{C}^{1}(\overline{\mathcal{V}})^{3}$ and $(u, \sigma, w) \in \mathcal{C}^{1}(\overline{\mathcal{V}})^{3 \times 9 \times 3}$, we deduce that

$$
\begin{aligned}
& \int_{\mathcal{V}_{0}} h \cdot(\nabla \hat{q} \delta x) \mathrm{d} x_{0}+\int_{\mathcal{V}}\left[\Phi(u, \sigma, w)\left(\operatorname{Idiv} \delta x-\nabla \delta x^{T}\right)\right. \\
& \left.\quad+D_{(u, \sigma, w)} \Phi(u, \sigma, w)(\nabla u \delta x, \nabla \sigma \delta x, \nabla w \delta x)\right]: \nabla \hat{q} \mathrm{~d} x \\
& =\int_{\partial \mathcal{V}_{0}}\left(h \otimes \delta x F^{-T} n_{0}\right) \cdot \hat{q} \mathrm{~d} a_{0} \\
& \quad+\int_{\partial \mathcal{V}}\left[D_{(u, \sigma, w)} \Phi(u, \sigma, w)(\nabla u \delta x, \nabla \sigma \delta x, \nabla w \delta x) n-\Phi(u, \sigma, w) \eta(\delta x)\right] \cdot \hat{q} \mathrm{~d} a, \\
& \forall \hat{q} \in C^{2}(\overline{\mathcal{V}})^{4}
\end{aligned}
$$


with $\eta(\delta x)=-\left(\operatorname{I} \operatorname{div} \delta x-\nabla \delta x^{T}\right) n$.

Proof. We transport the first term in (14) inside $\mathcal{V}$, and we get

$$
\begin{aligned}
& \int_{\mathcal{V}} {\left[\frac{1}{J} h \otimes \delta x+\Phi(u, \sigma, w)\left(\operatorname{I} \operatorname{div} \delta x-\nabla \delta x^{T}\right)\right.} \\
&\left.\quad+D_{(u, \sigma, w)} \Phi(u, \sigma, w)(\nabla u \delta x, \nabla \sigma \delta x, \nabla w \delta x)\right]: \nabla \hat{q} \mathrm{~d} x=0, \quad \forall \hat{q} \in C_{0}^{2}(\mathcal{V})^{4} .
\end{aligned}
$$

By setting,

$$
\begin{aligned}
G=\frac{1}{J} h \otimes \delta x+\Phi(u, \sigma, w)(\mathrm{I} \operatorname{div} \delta & \left.-\nabla \delta x^{T}\right) \\
& +D_{(u, \sigma, w)} \Phi(u, \sigma, w)(\nabla u \delta x, \nabla \sigma \delta x, \nabla w \delta x),
\end{aligned}
$$

we deduce that

$$
\operatorname{div} G=0, \quad \text { in } \mathcal{V} .
$$

which implies for $\hat{q} \in C^{2}(\overline{\mathcal{V}})^{4}$ the following,

$$
\int_{\mathcal{V}} G: \nabla \hat{q} \mathrm{~d} x=\int_{\partial \mathcal{V}} G n \cdot \hat{q} \mathrm{~d} a
$$

leading to (15).

Remark $9 \eta(\delta x)$ stands for the first order variation of the surface vector $-n \mathrm{~d} a$ and only depends on the trace of $\delta x$ on $\partial \mathcal{V}$.

We apply the last results to the particular reference problem (8). Thus, considering $\hat{v}^{s}=0$ and $\hat{v}^{f} \in \mathcal{C}_{0}^{2}\left(\Omega_{g}^{f}(t)\right)^{3}$ in (8), we get the following fluid subproblem:

$$
\begin{aligned}
\int_{\Omega_{0}^{f}} \frac{\partial J_{g} u_{g}}{\partial t}{\mid x_{0}}_{v_{1}} \cdot \hat{v}_{1}^{f} \mathrm{~d} x_{0}-\int_{\Omega_{g}^{f}(t)}\left[\phi\left(u_{g}, \sigma_{g}\right)-\mathrm{I}_{1} u_{g} \otimes w_{g}\right]: \nabla \hat{v}^{f} \mathrm{~d} x_{g}=0 \\
\forall \hat{v}^{f} \in \mathcal{C}_{0}^{2}\left(\Omega_{g}^{f}(t)\right)^{4}
\end{aligned}
$$

$\mathrm{RR} \mathrm{n}^{\circ} 4264$ 
Hence, we easily apply Lemma 4.1 with

$$
\begin{aligned}
\mathcal{V}_{0} & =\Omega_{0}^{f}, \\
x & =x_{g}^{f}, \\
\mathcal{V} & =\Omega_{g}^{f}(t), \\
h & =\mathrm{I}_{1}\left(J_{g} u_{g}\right) \\
\Phi(u, \sigma, w) & =\phi(u, \sigma)-\mathrm{I}_{1} u \otimes w .
\end{aligned}
$$

Then every state satisfying (16), satisfies also the following convected problem:

$$
\begin{aligned}
- & \int_{\Omega_{0}^{f}}\left(\frac{\partial J_{g} u_{g}}{\partial t} \otimes \delta x F_{g}^{-T}\right): \nabla_{0} \hat{v}_{1}^{f} \mathrm{~d} x_{0}-\int_{\Omega_{g}^{f}(t)}\left[\Phi(u, \sigma, w)\left(\mathrm{I} \operatorname{div} \delta x-\nabla \delta x^{T}\right)\right. \\
& +\Phi(u, \sigma, w)(\nabla u \delta x, \nabla \sigma \delta x, \nabla w \delta x)]: \nabla \hat{v} \mathrm{~d} x \\
= & -\int_{\Gamma_{0}^{s}}\left(\left.\frac{\partial J_{g} u_{g}}{\partial t}\right|_{\mid x_{0}} \otimes \delta x F_{g}^{-T} n_{0}\right) \cdot \hat{v}_{1} \mathrm{~d} a_{0} \\
& +\int_{\Gamma_{g}^{s}(t)}\left[\phi\left(u_{g}, \sigma_{g}\right)-\mathrm{I}_{1} u_{g} \otimes w_{g}\right] \eta(\delta x) \cdot \hat{v}^{f} \mathrm{~d} a_{g} \\
& -\int_{\Gamma_{g}^{s}(t)} D_{(u, \sigma)} \phi\left(u_{g}, \sigma_{g}\right)\left(\nabla u_{g} \delta x, \nabla \sigma_{g} \delta x\right) n_{g} \cdot \hat{v}^{f} \mathrm{~d} a_{g} \\
& +\int_{\Gamma_{g}^{s}(t)}\left(\nabla u_{g} \delta x \otimes w_{g}+u_{g} \otimes \nabla w_{g} \delta x\right) n_{g} \cdot \hat{v}_{1}^{f} \mathrm{~d} a_{g}, \quad \forall \hat{v}^{f} \in \mathcal{C}_{0}^{2}(\Omega)^{4} .
\end{aligned}
$$

Remark 10 The main advantage of this expression lies in turning volume integrals, with dependence on $\delta x$ over $\Omega_{g}^{f}(t)$, into surface integrals with dependence on $\delta x$.

\subsection{Linearization method}

The linearization process [5] consists of four steps:

1. recasting in the reference configuration $\Omega_{g}^{f}(t)$ of the convectif terms inside the perturbed problem, 
2. first order Taylor expansion in the perturbed problem using the definition $(12)$

3. cancellation of zero order terms by subtracting the reference problem (8),

4. simplification of first order terms, by subtracting the transported convected problem (17).

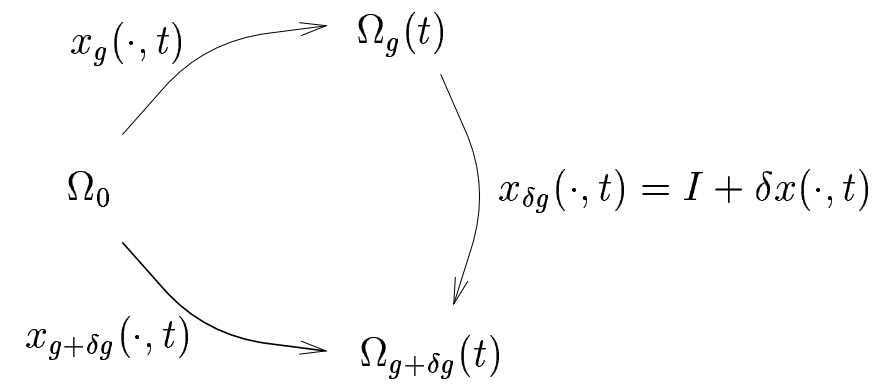

Figure 3: Mappings graph

Step 1: We set $F_{\delta g}=\nabla x_{\delta g}=\mathrm{I}+\nabla \delta x$ et $J_{\delta g}=\operatorname{det} F_{\delta g}$. After transport of the convectif terms, the problem (10) becomes:

$$
\begin{aligned}
& \int_{\Omega_{0}^{f}} \frac{\partial J_{g+\delta g} u_{g+\delta g}}{\partial t} \cdot \hat{v}_{1}^{f} \mathrm{~d} x_{0} \\
& \quad-\int_{\Omega_{g}^{f}(t)} J_{\delta g}\left[\phi\left(u_{g+\delta g}, \sigma_{g+\delta g}\right)-\mathrm{I}_{1}\left(u_{g+\delta g} \otimes w_{g+\delta g}\right)\right] F_{\delta g}^{-T}: \nabla \hat{v}^{f} \mathrm{~d} x_{g} \\
& \quad+\int_{\Omega_{0}^{s}} \ddot{x}_{g+\delta g}^{s} \cdot \hat{v}^{s} \mathrm{~d} x_{0}+\int_{\Omega_{0}^{s}} F_{g+\delta g} S_{g+\delta g}: \nabla_{0} \hat{v}^{s} \mathrm{~d} x_{0} \\
& \quad+\int_{\Gamma_{0}^{s}} J_{g+\delta g} \sigma_{g+\delta g} F_{g+\delta g}^{-T} n_{0} \cdot\left(\hat{v}^{s}-\hat{v}_{1}^{f}\right) \mathrm{d} a_{0} \\
& \quad+\int_{\Gamma_{g}^{s}(t)} J_{\delta g} \omega_{g+\delta g} \cdot\left(F_{\delta g}^{-T} n_{g}\right) \hat{v}_{2}^{f} \mathrm{~d} a_{g} \\
& =\int_{\Omega_{0}^{s}} f \cdot \hat{v}^{s} \mathrm{~d} a_{0}, \quad \forall \hat{v}=\left(\hat{v}^{s}, \hat{v}^{f}\right) \in \mathcal{C}_{0}^{2}\left(\Omega_{0}\right)^{3} \times \mathcal{C}_{0}^{2}(\Omega)^{4} .
\end{aligned}
$$

$\mathrm{RR} \mathrm{n}^{\circ} 4264$ 
Step 2: From the fluctuation definition (12) we obtain up to first order, the following identities (see appendix)

$$
\begin{aligned}
J_{g+\delta g} & =J_{g}(1+\operatorname{div} \delta x), \quad \text { in } \Omega_{0}, \\
F_{g+\delta g}^{-T} & =\left(\mathrm{I}-\nabla \delta x^{T}\right) F_{g}^{-T}, \quad \text { in } \Omega_{0}, \\
\sigma_{g+\delta g} & =\sigma_{g}+\nabla \sigma_{g} \delta x+\delta \sigma, \quad \text { in } \quad \Omega_{g}^{f}(t), \\
w_{g+\delta g} & =w_{g}+\delta w,
\end{aligned}
$$

with $\delta w$ et $\delta \sigma$ defined by

$$
\begin{aligned}
\delta w & =\left.\frac{\partial \delta x}{\partial t}\right|_{0}, \quad \text { in } \Omega . \\
\delta \sigma & =-\delta p \mathrm{I}+\nu\left[\nabla \delta u+(\nabla \delta u)^{T}\right], \quad \text { in } \quad \Omega_{g}^{f}(t) .
\end{aligned}
$$

We first linearize the terms involving time derivatives,

$$
\begin{aligned}
{\frac{\partial J_{g+\delta g} u_{g+\delta g}}{\partial t}}_{\mid x_{0}} & =\left.\frac{\partial}{\partial t}\right|_{\mid x_{0}}\left[J_{g}(1+\operatorname{div} \delta x)\left(u_{g}+\nabla u_{g} \delta x+\delta u\right)\right] \\
& =\left.\frac{\partial}{\partial t}\right|_{\mid x_{0}}\left\{J_{g}\left[(1+\operatorname{div} \delta x) u_{g}+\nabla u_{g} \delta x+\delta u\right]\right\} \\
& =\frac{\partial}{\partial t}{ }_{\mid x_{0}}\left[J_{g} u_{g}+J_{g} \operatorname{div}\left(u_{g} \otimes \delta x\right)+J_{g} \delta u\right] \\
& =\left.\frac{\partial}{\partial t}\right|_{x_{0}}\left[J_{g} u_{g}+\operatorname{div}_{0}\left(J_{g} u_{g} \otimes \delta x F_{g}^{-T}\right)+J_{g} \delta u\right]
\end{aligned}
$$


Since $\left(\dot{F_{g}^{-T}}\right)=-\nabla w_{g}^{T} F_{g}^{-T}$ and integrating by parts, we deduce

$$
\begin{aligned}
& \left.\int_{\Omega_{0}^{f}} \frac{\partial}{\partial t}\right|_{x_{0}}\left[\operatorname{div}_{0}\left(J_{g} u_{g} \otimes \delta x F_{g}^{-T}\right)\right] \cdot \hat{v}_{1}^{f} \mathrm{~d} x_{0} \\
& =\int_{\Omega_{0}^{f}} \operatorname{div}_{0}\left[\frac{\partial}{\partial t}\left(J_{x_{0}} u_{g} \otimes \delta x F_{g}^{-T}\right)\right] \cdot \hat{v}_{1}^{f} \mathrm{~d} x_{0} \\
& =\int_{\Omega_{0}^{f}} \operatorname{div}_{0}\left[\frac{\partial J_{g} u_{g}}{\partial t} \mid x_{0} \otimes \delta x F_{g}^{-T}+J_{g} u_{g} \otimes \delta w F_{g}^{-T}+J_{g} u_{g} \otimes \delta x\left(F_{g}^{-T}\right)\right] \cdot \hat{v}_{1}^{f} \mathrm{~d} x_{0} \\
& =-\int_{\Omega_{0}^{f}}\left(\frac{\partial J_{g} u_{g}}{\partial t} \mid x_{0} \otimes \delta x F_{g}^{-T}\right): \nabla_{0} \hat{v}_{1}^{f} \mathrm{~d} x_{0} \\
& \quad-\int_{\Omega_{g}^{f}(t)}\left[u_{g} \otimes \delta w-u_{g} \otimes\left(\nabla w_{g} \delta x\right)\right]: \nabla \hat{v}_{1}^{f} \mathrm{~d} x_{g} \\
& \quad+\int_{\Gamma_{0}^{s}}\left(\frac{\partial J_{g} u_{g}}{\partial t} \otimes \delta x F_{g}^{-T} n_{0}\right) \cdot \hat{v}_{1}^{f} \mathrm{~d} a_{0} \\
& \quad+\int_{\Gamma_{g}^{s}(t)}\left[u_{g} \otimes \delta w-u_{g} \otimes\left(\nabla w_{g} \delta x\right)\right] n_{g} \cdot \hat{v}_{1}^{f} \mathrm{~d} a_{g} .
\end{aligned}
$$

Then we get the following first order terms:

$$
\begin{aligned}
& \int_{\Omega_{0}^{f}} \frac{\partial J_{g+\delta g} u_{g+\delta g}}{\partial t} \cdot \hat{v}_{1}^{f} \mathrm{~d} x_{0}=\int_{\Omega_{0}^{f}} \frac{\partial J_{g}\left(u_{g}+\delta u\right)}{\partial t} \cdot x_{0} \\
& \quad-\int_{\Omega_{0}^{f}}^{f} \mathrm{~d} x_{0} \\
& \quad-\int_{\Omega_{g}^{f}(t)}\left[u_{g} \otimes \delta w-u_{g} \otimes\left(\nabla w_{g} \delta x\right)\right]: \nabla \hat{v}_{1}^{f} \mathrm{~d} x_{g} \\
& \quad+\int_{\Gamma_{0}^{s}}\left(\frac{\partial J_{g} u_{g}}{\partial t} \mid x_{0} F_{g}^{-T}\right): \nabla_{0} \hat{v}_{1}^{f} \mathrm{~d} x_{0} \\
& \quad+\int_{\Gamma_{g}^{s}(t)}\left[u_{g} \otimes \delta w-u_{g}^{-T} n_{0}\right) \cdot \hat{v}_{1}^{f} \mathrm{~d} a_{0}
\end{aligned}
$$

$\mathrm{RR} \mathrm{n}^{\circ} 4264$ 
Concerning the convectif terms, we have

$$
\begin{aligned}
& \int_{\Omega_{g}^{f}(t)} J_{\delta g} u_{g+\delta g} \otimes w_{g+\delta g} F_{\delta g}^{-T}: \nabla \hat{v}_{1}^{f} \mathrm{~d} x_{g}=\int_{\Omega_{g}^{f}(t)}\left(u_{g} \otimes w_{g}+\delta u \otimes w_{g}\right): \nabla \hat{v}_{1}^{f} \mathrm{~d} x_{g} \\
& +\int_{\Omega_{g}^{f}(t)}\left[\left(\nabla u_{g} \delta x\right) \otimes w_{g}+u_{g} \otimes \delta w+u_{g} \otimes w_{g}\left(\mathrm{I} \operatorname{div} \delta x-\nabla \delta x^{T}\right)\right]: \nabla \hat{v}_{1}^{f} \mathrm{~d} x_{g},
\end{aligned}
$$

and

$$
\begin{aligned}
& -\int_{\Omega_{g}^{f}(t)} J_{\delta g} \phi\left(u_{g+\delta g}, \sigma_{g+\delta g}\right) F_{\delta g}^{-T}: \nabla \hat{v}^{f} \mathrm{~d} x_{g} \\
& =-\int_{\Omega_{g}^{f}(t)}\left[\phi\left(u_{g}, \sigma_{g}\right)+D_{(u, \sigma)} \phi\left(u_{g}, \sigma_{g}\right)(\delta u, \delta \sigma)\right]: \nabla \hat{v}^{f} \mathrm{~d} x_{g} \\
& \quad-\int_{\Omega_{g}^{f}(t)}\left[\phi\left(u_{g}, \sigma_{g}\right)\left(\mathrm{I} \operatorname{div} \delta x-\nabla \delta x^{T}\right)\right. \\
& \left.\quad+D_{(u, \sigma)} \phi\left(u_{g}, \sigma_{g}\right)\left(\nabla u_{g} \delta x, \nabla \sigma_{g} \delta x\right)\right]: \nabla \hat{v}^{f} \mathrm{~d} x_{g} .
\end{aligned}
$$

Finally, the boundary contributions are reduced to

$$
\begin{gathered}
\int_{\Gamma_{0}^{s}} J_{g+\delta g} \sigma_{g+\delta g} F_{g+\delta g}^{-T} n_{0} \cdot\left(\hat{v}^{s}-\hat{v}_{1}^{f}\right) \mathrm{d} a_{0}=\int_{\Gamma_{0}^{s}} J_{g}\left(\sigma_{g}+\delta \sigma\right) F_{g}^{-T} n_{0} \cdot\left(\hat{v}^{s}-\hat{v}_{1}^{f}\right) \mathrm{d} a_{0} \\
\left.+\int_{\Gamma_{0}^{s}} J_{g}\left[\sigma_{g}\left(\operatorname{Idiv} \delta x-\nabla \delta x^{T}\right)+\nabla \sigma_{g} \delta x\right)\right] F_{g}^{-T} n_{0} \cdot\left(\hat{v}^{s}-\hat{v}_{1}^{f}\right) \mathrm{d} a_{0} . \quad(22)
\end{gathered}
$$

and

$$
\begin{aligned}
\int_{\Gamma_{g}^{s}(t)} J_{\delta g} \omega_{g+\delta g} \cdot\left(F_{\delta g}^{-T} n_{g}\right) \hat{v}_{2}^{f} \mathrm{~d} a_{g}= & \int_{\Gamma_{g}^{s}(t)} \omega_{g} \cdot n_{g} \hat{v}_{2}^{f} \mathrm{~d} a_{g} \\
& +\int_{\Gamma_{g}^{s}(t)}\left(\delta \omega \cdot n_{g}-\omega_{g} \cdot \eta(\delta x)\right) \hat{v}_{2}^{f} \mathrm{~d} a_{g}
\end{aligned}
$$

Step 3: We substitute in (18) the identities (19)-(23). After subtraction of the reference problem (8), we obtain 


$$
\begin{aligned}
& \int_{\Omega_{0}^{f}} \frac{\partial J_{g} \delta u}{\partial t} \cdot \hat{x}_{0}^{f} \mathrm{~d} x_{0}-\int_{\Omega_{g}^{f}(t)}\left[D_{u, \sigma} \phi\left(u_{g}, \sigma_{g}\right)(\delta u, \delta \sigma)-\mathrm{I}_{1} \delta u \otimes w_{g}\right]: \nabla \hat{v}^{f} \mathrm{~d} x_{g} \\
& -\int_{\Omega_{0}^{f}}\left(\frac{\partial J_{g} u_{g}}{\partial t} \mid x_{0} \otimes \delta x F_{g}^{-T}\right): \nabla_{0} \hat{v}_{1}^{f} \mathrm{~d} x_{0}+\int_{\Omega_{g}^{f}(t)} u_{g} \otimes\left(\nabla \omega_{g} \delta x\right): \nabla \hat{v}_{1}^{f} \mathrm{~d} x_{g} \\
& -\int_{\Omega_{g}^{f}(t)}\left[D_{(u, \sigma)} \phi\left(u_{g}, \sigma_{g}\right)\left(\nabla u_{g} \delta x, \nabla \sigma_{g} \delta x\right)-\mathrm{I}_{1}\left(\nabla u_{g} \delta x\right) \otimes \omega_{g}\right]: \nabla \hat{v}^{f} \mathrm{~d} x_{g} \\
& -\int_{\Omega_{g}^{f}(t)}\left[\left(\phi\left(u_{g}, \sigma_{g}\right)-\mathrm{I}_{1} u_{g} \otimes \omega_{g}\right)\left(\mathrm{I} \mathrm{div} \delta x-\nabla \delta x^{T}\right)\right]: \nabla \hat{v}^{f} \mathrm{~d} x_{g} \\
& +\int_{\Gamma_{0}^{s}}\left(\frac{\partial J_{g} u_{g}}{\partial t} \otimes \delta x F_{g}^{-T} n_{g}\right) \cdot \hat{v}_{1}^{f} \mathrm{~d} a_{0} \\
& +\int_{\Gamma_{g}^{s}(t)}\left[u_{g} \otimes \delta w-u_{g} \otimes\left(\nabla w_{g} \delta x\right)\right] n_{g} \cdot \hat{v}_{1}^{f} \mathrm{~d} a_{g} \\
& +\int_{\Gamma_{g}^{s}(t)}\left(-\sigma_{g} \eta(\delta x)+\nabla \sigma_{g} \delta x n_{g}+\delta \sigma n_{g}\right) \cdot\left(\hat{v}^{s}-\hat{v}_{1}^{f}\right) \mathrm{d} a_{g} \\
& +\int_{\Gamma_{g}^{s}(t)}\left(\delta \omega \cdot n_{g}-\omega_{g} \cdot \eta(\delta x)\right) \hat{v}_{2}^{f} \mathrm{~d} a_{g}+\int_{\Omega_{0}^{s}} \delta \ddot{x}^{s} \cdot \hat{v}^{s} \mathrm{~d} x_{0} \\
& +\int_{\Omega_{0}^{s}}\left(\nabla_{0} \delta x S_{g}+F_{g} \delta S\right): \nabla \hat{v}^{s} \mathrm{~d} x_{0}=0, \forall \hat{v}=\left(\hat{v}^{s}, \hat{v}^{f}\right) \in \mathcal{C}_{0}^{2}\left(\Omega_{0}\right)^{3} \times \mathcal{C}_{0}^{2}(\Omega)^{3},
\end{aligned}
$$

where $\delta S=S^{\prime}\left(x_{g}^{s}\right) \delta x^{s}=\frac{\partial S}{\partial x}\left(x_{g}^{s}\right) \delta x^{s}$, which represents the solid constitutive law linearization around the state $x_{g}^{s}$.

$\mathrm{RR} \mathrm{n}^{\circ} 4264$ 
By setting $\Phi(u, \sigma, \omega)=\phi(u, \sigma)-\mathrm{I}_{1} u \otimes \omega$, we get the following expression

$$
\begin{aligned}
& \int_{\Omega_{0}^{f}} \frac{\partial J_{g} \delta u}{\partial t}{ }_{\mid x_{0}} \cdot \hat{v}_{1}^{f} \mathrm{~d} x_{0}-\int_{\Omega_{g}^{f}(t)}\left[D_{u, \sigma} \phi\left(u_{g}, \sigma_{g}\right)(\delta u, \delta \sigma)-\mathrm{I}_{1} \delta u \otimes w_{g}\right]: \nabla \hat{v}^{f} \mathrm{~d} x_{g} \\
& -\int_{\Omega_{0}^{f}}\left({\frac{\partial J_{g} u_{g}}{\partial t}}_{\mid x_{0}} \otimes \delta x F_{g}^{-T}\right): \nabla_{0} \hat{v}_{1}^{f} \mathrm{~d} x_{0} \\
& +\int_{\Gamma_{g}^{s}(t)}\left(-\sigma_{g} \eta(\delta x)+\nabla \sigma_{g} \delta x n_{g}+\delta \sigma n_{g}\right) \cdot\left(\hat{v}^{s}-\hat{v}_{1}^{f}\right) \mathrm{d} a_{g} \\
& -\int_{\Omega_{g}^{f}(t)}\left[\Phi\left(u_{g}, \sigma_{g}, w_{g}\right)\left(\mathrm{I} \operatorname{div} \delta x-\nabla \delta x^{T}\right)\right. \\
& \left.+D_{(u, \sigma, w)} \Phi\left(u_{g}, \sigma_{g}, w_{g}\right)\left(\nabla u_{g} \delta x, \nabla \sigma_{g} \delta x, \nabla w_{g} \delta x\right)\right]: \nabla \hat{v}^{f} \mathrm{~d} x_{g} \\
& +\int_{\Gamma_{0}^{s}}\left(\frac{\partial J_{g} u_{g}}{\partial t}{ }_{\mid x_{0}} \otimes \delta x F_{g}^{-T} n_{g}\right) \cdot \hat{v}_{1}^{f} \mathrm{~d} a_{0} \\
& +\int_{\Gamma_{g}^{s}(t)}\left[u_{g} \otimes \delta w-u_{g} \otimes\left(\nabla w_{g} \delta x\right)\right] n_{g} \cdot \hat{v}_{1}^{f} \mathrm{~d} a_{g} \\
& +\int_{\Gamma_{g}^{s}(t)}\left(\delta \omega \cdot n_{g}-\omega_{g} \cdot \eta(\delta x)\right) \hat{v}_{2}^{f} \mathrm{~d} a_{g}+\int_{\Omega_{0}^{s}} \delta \ddot{x^{s}} \cdot \hat{v}^{s} \mathrm{~d} x_{0} \\
& +\int_{\Omega_{0}^{s}}\left(\nabla_{0} \delta x S_{g}+F_{g} \delta S\right): \nabla_{0} \hat{v}^{s} \mathrm{~d} x_{0}=0, \quad \forall \hat{v}=\left(\hat{v}^{s}, \hat{v}^{f}\right) \in \mathcal{C}_{0}^{2}\left(\Omega_{0}\right)^{3} \times \mathcal{C}_{0}^{2}(\Omega)^{3} .
\end{aligned}
$$


Step 4: Then we substract the convected problem (17) to the last expression and we get

$$
\begin{aligned}
& \int_{\Omega_{0}^{f}} \frac{\partial J_{g} \delta u}{\partial t} \cdot \hat{v}_{1}^{f} \mathrm{~d} x_{0}-\int_{\Omega_{g}^{f}(t)}\left[D_{(u, \sigma)} \phi\left(u_{g}, \sigma_{g}\right)(\delta u, \delta \sigma)-\mathrm{I}_{1} \delta u \otimes w_{g}\right]: \nabla \hat{v}^{f} \mathrm{~d} x_{g} \\
& +\int_{\Gamma_{g}^{s}(t)}\left(-\sigma_{g} \eta(\delta x)+\nabla \sigma_{g} \delta x n_{g}+\delta \sigma n_{g}\right) \cdot\left(\hat{v}^{s}-\hat{v}_{1}^{f}\right) \mathrm{d} a_{g} \\
& +\int_{\Gamma_{g}^{s}(t)}\left[u_{g} \otimes \delta w+\left(\nabla u_{g} \delta x\right) \otimes w_{g}\right] n_{g} \cdot \hat{v}_{1}^{f} \mathrm{~d} a_{g}+\int_{\Gamma_{g}^{s}(t)}\left(\delta \omega \cdot n_{g}-\omega_{g} \cdot \eta(\delta x)\right) \hat{v}_{2}^{f} \mathrm{~d} a_{g} \\
& +\int_{\Gamma_{g}^{s}(t)}\left[\left(\phi\left(u_{g}, \sigma_{g}\right)-\mathrm{I}_{1} u_{g} \otimes w_{g}\right) \eta(\delta x)\right. \\
& \left.\quad-D_{(u, \sigma)} \phi\left(u_{g}, \sigma_{g}\right)\left(\nabla u_{g} \delta x, \nabla \sigma_{g} \delta x\right) n_{g}\right] \cdot \hat{v}^{f} \mathrm{~d} a_{g} \\
& +\int_{\Omega_{0}^{s}} \delta \ddot{x}^{s} \cdot \hat{v}^{s} \mathrm{~d} x_{0}+\int_{\Omega_{0}^{s}}\left(\nabla_{0} \delta x S_{g}+F_{g} \delta S\right): \nabla_{0} \hat{v}^{s} \mathrm{~d} x_{0}=0, \\
& \forall \hat{v}=\left(\hat{v}^{s}, \hat{v}^{f}\right) \in \mathcal{C}_{0}^{2}\left(\Omega_{0}\right)^{3} \times \mathcal{C}_{0}^{2}(\Omega)^{4} .
\end{aligned}
$$

From (12), (9) et (11), we obtain the following boundary conditions:

$$
\begin{aligned}
\delta u & =\delta g, \quad \text { on } \Gamma_{0}^{f}, \\
\delta u & =\delta w-\nabla u_{g} \delta x, \text { on } \Gamma_{g}^{s}(t) .
\end{aligned}
$$

Remark 11 The transpiration condition (25) differs from the one obtained in Fanion-Fernández-Le Tallec [5], from the fact that it is written on a moving interface since the reference problem is unsteady.

$\mathrm{RR} \mathrm{n}^{\circ} 4264$ 
Furthermore, using that on $\Gamma_{g}^{s}(t)$ we have $w_{g}=u_{g}$, et from (25), the surface integrals (24) can be simplified and we get

$$
\begin{aligned}
& \int_{\Omega_{0}^{f}} \frac{\partial J_{g} \delta u}{\partial t} \cdot \hat{x}_{0} \\
& +\int_{\Gamma_{g}^{s}(t)}\left(-\sigma_{g} \eta(\delta x)+\nabla x_{g} \delta x n_{g}+\delta \sigma n_{g}\right) \cdot \hat{v}^{s} \mathrm{~d} a_{g}+\int_{\Gamma_{g}^{s}(t)}\left(D_{g} \otimes \delta u-\delta \sigma\right) n_{g} \cdot \hat{v}_{1}^{f} \mathrm{~d} a_{g} \\
& +\int_{\Gamma_{g}^{s}(t)} \delta u \cdot n_{g} \hat{v}_{2}^{f} \mathrm{~d} a_{g}+\int_{\Omega_{0}^{s}} \delta \ddot{x}^{s} \cdot \hat{v}^{s} \mathrm{~d} x_{0}+\int_{\Omega_{0}^{s}}\left(\nabla_{0} \delta x S_{g}+F_{g} \delta S\right): \nabla_{0} \hat{v}^{s} \mathrm{~d} x_{0}=0, \\
& \forall \hat{v}=\left(\hat{v}^{s}, \hat{v}^{f}\right) \in \mathcal{C}_{0}^{2}\left(\Omega_{0}\right)^{3} \times \mathcal{C}_{0}^{2}(\Omega)^{3} .
\end{aligned}
$$

On $\Gamma_{g}^{s}(t)$, we have also the following identity,

$$
u_{g} \otimes \delta u-\delta \sigma=\delta u \otimes u_{g}+u_{g} \otimes \delta u-\delta \sigma-\delta u \otimes \omega_{g},
$$

which allows us to write

$$
\begin{aligned}
& \int_{\Gamma_{g}^{s}(t)}\left(u_{g} \otimes \delta u-\delta \sigma\right) n_{g} \cdot \hat{v}_{1}^{f} \mathrm{~d} a_{g}+\int_{\Gamma_{g}^{s}(t)} \delta u \cdot n_{g} \hat{v}_{2}^{f} \mathrm{~d} a_{g} \\
& =\int_{\Gamma_{g}^{s}(t)}\left[\mathrm{I}_{1}\left(u_{g} \otimes \delta u-\delta \sigma\right)+\mathrm{I}_{2} \otimes \delta u\right] n_{g} \cdot \hat{v}^{f} \\
& =\int_{\Gamma_{g}^{s}(t)}\left[D_{(u, \sigma)} \phi\left(u_{g}, \sigma_{g}\right)(\delta u, \delta \sigma)-\mathrm{I}_{1} \delta u \otimes w_{g}\right] n_{g} \cdot \hat{v}^{f} \mathrm{~d} a_{g} .
\end{aligned}
$$


Using this last identity in (26), we may obtain

$$
\begin{aligned}
& \int_{\Omega_{0}^{f}} \frac{\partial J_{g} \delta u}{\partial t} \cdot \hat{x}_{0}^{f} \mathrm{~d} x_{0}-\int_{\Omega_{g}^{f}(t)}\left[D_{(u, \sigma)} \phi\left(u_{g}, \sigma_{g}\right)(\delta u, \delta \sigma)-\mathrm{I}_{1} \delta u \otimes w_{g}\right]: \nabla \hat{v}^{f} \mathrm{~d} x_{g} \\
& +\int_{\Gamma_{g}^{s}(t)}\left(-\sigma_{g} \eta(\delta x)+\nabla \sigma_{g} \delta x n_{g}+\delta \sigma n_{g}\right) \cdot \hat{v}^{s} \mathrm{~d} a_{g} \\
& +\int_{\Gamma_{g}^{s}(t)}\left[D_{(u, \sigma)} \phi\left(u_{g}, \sigma_{g}\right)(\delta u, \delta \sigma)-\mathrm{I}_{1} \delta u \otimes w_{g}\right] n_{g} \cdot \hat{v}^{f} \mathrm{~d} a_{g} \\
& +\int_{\Omega_{0}^{s}} \delta \ddot{x}^{s} \cdot \hat{v}^{s} \mathrm{~d} x_{0}+\int_{\Omega_{0}^{s}}\left(\nabla_{0} \delta x S_{g}+F_{g} \delta S\right): \nabla_{0} \hat{v}^{s} \mathrm{~d} x_{0}=0, \\
& \forall \hat{v}=\left(\hat{v}^{s}, \hat{v}^{f}\right) \in \mathcal{C}_{0}^{2}\left(\Omega_{0}\right)^{3} \times \mathcal{C}_{0}^{2}(\Omega)^{4}
\end{aligned}
$$

As a final step, we may transport the terms involving time derivative in $\Omega_{g}^{f}(t)$ and we obtain that $(\delta u, \delta p, \delta x)$ satisfies the following linear variational problem:

$$
\begin{aligned}
& \int_{\Omega_{g}^{f}(t)} \frac{\partial \delta u}{\partial t} x_{g} \cdot \hat{v}_{1}^{f} \mathrm{~d} x_{0}-\int_{\Omega_{g}^{f}(t)}\left[D_{(u, \sigma)} \phi\left(u_{g}, \sigma_{g}\right)(\delta u, \delta \sigma)\right]: \nabla \hat{v}^{f} \mathrm{~d} x_{g} \\
& +\int_{\Gamma_{g}^{s}(t)}\left(-\sigma_{g} \eta(\delta x)+\nabla \sigma_{g} \delta x n_{g}+\delta \sigma n_{g}\right) \cdot \hat{v}^{s} \mathrm{~d} a_{g} \\
& +\int_{\Gamma_{g}^{s}(t)}\left[D_{(u, \sigma)} \phi\left(u_{g}, \sigma_{g}\right)(\delta u, \delta \sigma)\right] n_{g} \cdot \hat{v}^{f} \mathrm{~d} a_{g}+\int_{\Omega_{0}^{s}} \ddot{\delta} \ddot{x}^{s} \cdot \hat{v}^{s} \mathrm{~d} x_{0} \\
& +\int_{\Omega_{0}^{s}}\left(\nabla_{0} \delta x S_{g}+F_{g} \delta S\right): \nabla_{0} \hat{v}^{s} \mathrm{~d} x_{0}=0, \quad \forall \hat{v}=\left(\hat{v}^{s}, \hat{v}^{f}\right) \in \mathcal{C}_{0}^{2}\left(\Omega_{0}\right)^{3} \times \mathcal{C}_{0}^{2}(\Omega)^{3} .
\end{aligned}
$$

This completes the determination of the linearized problem which may characterize the variation of the reference problem (8) with respect to perturbations of the Dirichlet boundary conditions.

In order to link this result with the coupled strong ALE formulation (5), it is needed to perform integrations by parts inside (27). This may lead to the linearized problem in strong formulation. As we shall see in the following, this

$\mathrm{RR} \mathrm{n}^{\circ} 4264$ 
formulation consists of two subproblems satisfied respectively by $(\delta u, \delta p)$ in $\Omega_{g}^{s}(t)$ and $\delta x^{s}$ in $\Omega_{0}^{s}$. These two subsystems are coupled through non-standard boundary conditions on $\Gamma_{g}^{s}(t)$ :

- setting $\hat{v}^{s}=0$ in (27) and after integration by parts, we obtain the linearized fluid subproblem:

$$
\begin{array}{r}
\left.\frac{\partial \delta u}{\partial t}\right|_{g}+\operatorname{div}\left(\delta u \otimes u_{g}+u_{g} \otimes \delta u-\delta \sigma\right)=0, \quad \text { in } \quad \Omega_{g}^{f}(t), \\
\operatorname{div} \delta u=0, \text { in } \Omega_{g}^{f}(t), \\
\delta u=\dot{\delta}-\nabla u_{g} \delta x, \text { on } \Gamma_{g}^{s}(t), \\
\delta u=\delta g, \text { on } \Gamma_{g}^{f}(t),
\end{array}
$$

with $\delta \sigma=-\delta p \mathrm{I}+\nu\left(\nabla \delta u+\nabla \delta u^{T}\right)$.

- setting $\hat{v}^{f}=0$ in (27) and after integration by parts, we obtain the linearized solid subproblem:

$$
\begin{aligned}
& \ddot{\delta x^{s}}-\operatorname{div}_{0}\left[F_{g} \delta S+\nabla_{0} \delta x^{s} S_{g}\right]=0, \quad \text { in } \quad \Omega_{0}^{s}, \\
& {\left[F_{g} \delta S+\nabla_{0} \delta x S_{g}\right] n_{0}=J_{g}\left\|F_{g}^{-T} n_{0}\right\|\left(-\sigma_{g} \eta\left(\delta x^{s}\right)\right.} \\
& \left.+\nabla \sigma_{g} \delta x^{s} n_{g}+\delta \sigma n_{g}\right) \text {, on } \Gamma_{0}^{s} \text {. }
\end{aligned}
$$

with $\left(\delta u, \delta x^{s}, \dot{\delta x^{s}}\right)_{\mid t=0}=0$ in $\Omega_{g}^{f}(0) \times\left(\Omega_{0}^{s}\right)^{2}$.

\subsection{State derivative computation}

As we mentioned in (12), the fluctuation around $\left(u_{g}, p_{g}, x_{g}\right)$ are given by

$$
\begin{aligned}
x_{g+\delta g} & =x_{g}+\delta x, \quad \text { in } \Omega_{0}, \\
u_{g+\delta g}\left(I_{\Omega_{g}^{f}(t)}+\delta x\right) & =u_{g}+\nabla u_{g} \delta x+\delta u, \quad \text { in } \Omega_{g}^{f}(t), \\
p_{g+\delta g}\left(I_{\Omega_{g}^{f}(t)}+\delta x\right) & =p_{g}+\nabla p_{g} \delta x+\delta p, \quad \text { in } \Omega_{g}^{f}(t) .
\end{aligned}
$$


The first order terms correspond to the state derivative around $\left(u_{g}, p_{g}, x_{g}\right)$ in the direction perturbation $\delta g$, i.e

$$
\begin{aligned}
& \frac{D u_{g}}{D g}=\delta u+\nabla u_{g} \delta x^{f}, \quad \text { in } \quad \Omega_{g}^{f}(t) \\
& \frac{D p_{g}}{D g}=\delta p+\nabla p_{g} \delta x^{f}, \quad \text { in } \quad \Omega_{g}^{f}(t) \\
& \frac{D x_{g}}{D g}=\delta x, \quad \text { in } \quad \Omega_{0} .
\end{aligned}
$$

The solution of the linearized problem (27) provides the fluctuations $\left(\delta u, \delta p, \delta x^{s}\right)$, and $\delta x^{f}$ can be deduced from $\delta x^{s}$ through the following expression:

$$
\delta x^{f}=\frac{D x_{g}^{f}}{D g} \delta g=\frac{D \operatorname{Ext}\left(x_{g}^{s}\right)}{D g} \delta g=\operatorname{Ext}^{\prime}\left(x_{s}^{g}\right)\left(\frac{D x_{g}^{s}}{D g} \delta g\right)=\operatorname{Ext}^{\prime}\left(x_{g}^{s}\right) \delta x^{s} .
$$

Finally, the state derivatives are given by the following expressions:

$$
\begin{aligned}
& \frac{D u_{g}}{D g}=\delta u+\nabla u_{g} \operatorname{Ext}^{\prime}\left(x_{g}^{s}\right) \delta x^{s}, \quad \text { in } \Omega_{g}^{f}(t), \\
& \frac{D p_{g}}{D g}=\delta p+\nabla p_{g} \operatorname{Ext}^{\prime}\left(x_{g}^{s}\right) \delta x^{s}, \quad \text { in } \Omega_{g}^{f}(t), \\
& \frac{D x_{g}^{s}}{D g}=\delta x^{s}, \quad \text { in } \quad \Omega_{0} .
\end{aligned}
$$

with $\left(\delta u, \delta p, \delta x^{s}\right)$ satisfying the linearized system(27).

Remark 12 In case the operator Ext is linear, the fluid state derivative can be reduced to:

$$
\begin{aligned}
& \frac{D u_{g}}{D g}=\delta u+\nabla u_{g} \operatorname{Ext}\left(\delta x^{s}\right), \quad \text { in } \quad \Omega_{g}^{f}(t), \\
& \frac{D p_{g}}{D g}=\delta p+\nabla p_{g} \operatorname{Ext}\left(\delta x^{s}\right), \quad \text { in } \quad \Omega_{g}^{f}(t) .
\end{aligned}
$$

$\operatorname{RR} n^{\circ} 4264$ 


\section{Conclusion and perspectives}

In this article, we have shown how to compute the derivative with respect to Dirichlet boundary conditions of a coupled fluid-structure state. This derivative is obtained by solving two, fluid and solid, subproblems coupled through non-standard transpiration boundary conditions at the fluid-structure interface of the reference coupled system.

This result is a first step towards the analysis of optimal control problems for fluid-structure interaction systems, where we cannot neglect the displacement of the interface. This problem will be addressed in a forthcoming paper [16].

Concerning the numerical approximation of the linearized problem, it would be of great interest to perform the linearization process on the discretized fluidstructure coupled system. This question is currently under investigation.

\section{Appendix}

In this section, we shall state the proof of different identities used in the proof of the main result of this article.

We first recall basic differential calculus results [2] without quoting there proof.

Lemma 2 The map $\operatorname{det}: A \longrightarrow \operatorname{det} A$, acting on the set of non-singular matrices is differentiable. Moreover,

$$
\frac{D \operatorname{det}}{D A}(A)(U)=\operatorname{det} A \operatorname{tr}\left(U A^{-1}\right), \quad \forall U \in \mathbb{R}^{3 \times 3} .
$$

Lemma 3 The map $A \longrightarrow A^{-1}$, acting on the set of non-singular matrices is differentiable. Moreover,

$$
\frac{D A^{-1}}{D A}(U)=-A^{-1} U A^{-1}, \quad \forall U \in \mathbb{R}^{3 \times 3} .
$$


Using the notation used in the article, we consider two regular, injective maps

$$
\begin{gathered}
x_{g}: \Omega_{0} \times \mathbb{R}^{+} \longrightarrow \mathbb{R}^{3}, \\
x_{g+\delta g}: \Omega_{0} \times \mathbb{R}^{+} \longrightarrow \mathbb{R}^{3},
\end{gathered}
$$

see figure 3. Setting

$$
\delta x=x_{g+\delta g}-x_{g} .
$$

Lemma 4 Up to first order in $\delta x$, we have the following identities:

$$
\begin{aligned}
J_{g+\delta g} & =J_{g}(1+\operatorname{div} \delta x), \quad \text { in } \quad \Omega_{0} \\
F_{g+\delta g}^{-1} & =F_{g}^{-1}(\mathrm{I}-\nabla \delta x), \quad \text { in } \quad \Omega_{0} \\
\sigma_{g+\delta g} & =\sigma_{g}+\nabla \sigma_{g} \delta x+\delta \sigma, \quad \text { in } \quad \Omega_{g}^{f}(t) .
\end{aligned}
$$

Proof. In order to get (28), we shall perform a first order Taylor expansion using lemma 2. Hence,

$$
\begin{aligned}
J_{g+\delta g} & =\operatorname{det} F_{g+\delta g} \\
& =\operatorname{det}\left(F_{g}+\nabla_{0} \delta x\right) \\
& =\operatorname{det} F_{g}+\operatorname{det} F_{g} \operatorname{tr}\left(\nabla_{0} \delta x F_{g}^{-1}\right) \\
& =J_{g}(1+\operatorname{tr}(\nabla \delta x)) \\
& =J_{g}(1+\operatorname{div} \delta x) .
\end{aligned}
$$

We may obtain (29) from lemma 3. Then,

$$
\begin{aligned}
F_{g+\delta g}^{-1} & =\left(F_{g}+\nabla_{0} \delta x\right)^{-1} \\
& =F_{g}^{-1}-F_{g}^{-1} \nabla_{0} \delta x F_{g}^{-1} \\
& =F_{g}^{-1}\left(\mathrm{I}-\nabla_{0} \delta x F_{g}^{-1}\right) \\
& =F_{g}^{-1}(\mathrm{I}-\nabla \delta x) .
\end{aligned}
$$

$\mathrm{RR} \mathrm{n}^{\circ} 4264$ 
For (30), using (12), we get

$$
\begin{aligned}
\sigma_{g+\delta g}= & -p_{g+\delta g} \mathrm{I}+\nu\left(\nabla u_{g+\delta g} F_{\delta g}{ }^{-1}+F_{\delta g}{ }^{T} \nabla u_{g+\delta g}{ }^{T}\right) \\
= & -\left(p_{g}+\nabla p_{g} \cdot \delta x+\delta p\right) \mathrm{I}+\nu\left[\nabla\left(u_{g}+\nabla u_{g} \delta x+\delta u\right)(\mathrm{I}-\nabla \delta x)\right. \\
& \left.+\left(\mathrm{I}-\nabla \delta x^{T}\right) \nabla\left(u_{g}+\nabla u_{g} \delta x+\delta u\right)^{T}\right] \\
= & -\nabla p_{g} \cdot \delta x \mathrm{I}+\nu\left[\nabla\left(\nabla u_{g} \delta x\right)-\nabla u_{g} \nabla \delta x+\nabla\left(\nabla u_{g} \delta x\right)^{T}-\nabla \delta x^{T} \nabla u_{g}{ }^{T}\right] \\
& +\sigma_{g}+\delta \sigma \\
= & \sigma_{g}-\nabla p_{g} \cdot \delta x \mathrm{I}+\nu\left[\nabla\left(\nabla u_{g}\right) \delta x+\nabla\left(\nabla u_{g}^{T}\right) \delta x\right]+\delta \sigma \\
= & \sigma_{g}+\nabla \sigma_{g} \delta x+\delta \sigma,
\end{aligned}
$$

which completes the proof.

\section{References}

[1] F. Abergel and R. Temam. On some control problems in fluid mechanics. Theoretical and Computational Fluid Dynamics, 1:303-325, 1990.

[2] P.G. Ciarlet. Mathematical elasticity. Vol. I. Three-dimensional elasticity. North-Holland Publishing, Amsterdam, 1988.

[3] C. Conca, J. San Martín, and M. Tucsnak. Existence of solutions for the equations modelling the motion of a rigid body in a viscous fluid. Comm. Partial Differential Equations, 25(5-6):1019-1042, 2000.

[4] B. Desjardins and M.J. Esteban. Existence of solutions for a model of fluid-rigid structure interaction. Arch. for Rat. Mech. Anal., 146:59-71, 1999 .

[5] T. Fanion, M.A. Fernández, and P. Le Tallec. Deriving adequate formulations for fluid-structure interactions problems: from ALE to transpiration. Rév. Européenne Élém. Finis, 9(6-7):681-708, 2000.

[6] M.A. Fernández and M. Moubachir. Étude de sensibilité d'un système mécanique en interaction fluide-structure. C. R. Acad. Sci. Paris, Série I, 333:487-492, 2001. 
[7] F. Flori and P. Orenga. Analysis of a nonlinear fluid-structure interaction problem in velocity-displacement formulation. Nonlinear Analysis, 35:561-587, 1999.

[8] C. Grandmont and Y. Maday. Fluid-structure interaction: A theoretical point of view. Rev. Européenne Élém. Finis, 9(6-7):633-653, 2001.

[9] M.D. Gunzburger, L. Hou, and T.P. Svobodny. Boundary velocity control of incompressible flow with application to viscous drag reduction . SIAM Journal of Control and Optimization, 30(1):167-181, 1992.

[10] M.D. Gunzburger, H.C. Lee, and G.A. Seregin. Global existence of weak solutions for viscous incompressible flows around a moving rigid body in three dimensions. J. math. fluid mech, 2(3):219-266, 2000.

[11] L.S. Hou and Y. Yan. Dynamics for controlled Navier-Stokes systems with distributed controls. SIAM Journal of Control and Optimization, 35(2):654-677, 1997.

[12] P. Le Tallec. Numerical methods for nonlinear three-dimensional elasticity. In Handbook of numerical analysis, Vol. III, pages 465-622. NorthHolland, Amsterdam, 1994.

[13] P. Le Tallec and J. Mouro. Fluid structure interaction with large structural displacements. Comput. Methods Appl. Mech. Engrg., 190(2425):3039-3067, 2001.

[14] A. Maruoka, M. Marin, and M. Kawahara. Optimal control in NavierStokes equations. International Journal of Computational Fluid Dynamics, 9:313-322, 1998.

[15] B. Mohammadi. Contrôle d'instationnarités en couplage fluide-structure. C. R. Acad. Sci., Paris, Ser. II, Fasc. b, Mec. Phys. Astron., 327(1):115$118,1999$.

[16] M. Moubachir and J.P. Zolesio. Identification of boundary conditions for a nonlinear unsteady fluid-structure interaction system. 2002. To appear.

$\mathrm{RR} \mathrm{n}^{\circ} 4264$ 
[17] C.M. Murea and Y. Maday. Existence of an optimal control for a nonlinear fluid-cable interaction problem. Technical report, CEMRACS, C.I.R.M, Luminy, France, 1996.

[18] A. Osses and J.P. Puel. Approximate controllability for a linear model of fluid-structure interaction. ESAIM: Optimisation and Calculus of Variations, 4:497-513, 1999.

[19] S. Piperno and C. Farhat. Design of efficient partitioned procedures for the transient solution of aeroelastic problems. Rev. Eur. Elém. Finis, 9(6-7):655-680, 2001.

[20] K.E. Willcox. Reduced-order aerodynamic models for aeroelastic control of turbomachines. PhD thesis, MIT, Department of Aeronautics and Astronautics, 2000. 


\section{Contents}

1 Introduction 3

2 Mechanical problem $\quad 4$

2.1 Coupled strong ALE formulation ........... 6

$\begin{array}{lll}3 & \text { Sensitivity analysis } & 7\end{array}$

4 Proof of the main result 9

4.1 Weak formulation . . . . . . . . . . . . . . 9

4.2 Transported reference problem . . . . . . . . . . . . . 12

4.3 Linearization method . . . . . . . . . . . 16

4.4 State derivative computation . . . . . . . . . . 26

5 Conclusion and perspectives $\quad 28$

$\mathrm{RR} \mathrm{n}^{\circ} 4264$ 


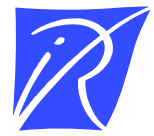

Unité de recherche INRIA Rocquencourt Domaine de Voluceau - Rocquencourt - BP 105 - 78153 Le Chesnay Cedex (France)

Unité de recherche INRIA Lorraine : LORIA, Technopôle de Nancy-Brabois - Campus scientifique 615, rue du Jardin Botanique - BP 101 - 54602 Villers-lès-Nancy Cedex (France)

Unité de recherche INRIA Rennes : IRISA, Campus universitaire de Beaulieu - 35042 Rennes Cedex (France)

Unité de recherche INRIA Rhône-Alpes : 655, avenue de l'Europe - 38330 Montbonnot-St-Martin (France)

Unité de recherche INRIA Sophia Antipolis : 2004, route des Lucioles - BP 93 - 06902 Sophia Antipolis Cedex (France)

INRIA - Domaine de Voluceau - Rocquencourt, BP 105 - 78153 Le Chesnay Cedex (France)

http://www.inria.fr

ISSN 0249-6399 